\title{
Intraductal pancreatic stent fragmentation in patients with walled-off pancreatic necrosis and pancreatic duct disruption
}

\author{
Mateusz Jagielski, Marian Smoczyński, Krystian Adrych \\ Department of Gastroenterology and Hepatology, Medical University of Gdansk, Gdansk, Poland
}

Gastroenterology Rev 2018; 13 (2): 157-159

DOI: https://doi.org/10.5114/pg.2018.75279

Address for correspondence: Mateusz Jagielski MD, PhD, Department of Gastroenterology and Hepatology, Medical University of Gdansk, 17 Smoluchowskiego St, 80-214 Gdansk, Poland, phone: +48 7811436 96, e-mail: matjagiel@gmail.com

We published our article in the November-December 2017 issue of "Digestive Endoscopy" [1]. In it we presented the results of treatment of a rare complication of pancreatic endotherapy in the form of intraductal stent fragmentation in 33 patients with chronic pancreatitis [1]. We demonstrated that most of the broken pancreatic stent fragments can be removed endoscopically from the pancreatic duct with an acceptable complication rate [1]. Furthermore, intraductal pancreatic stent fragmentation was discovered significantly more often in patients with chronic calcific pancreatitis and in patients with pancreatic stones inside the main pancreatic duct [1]. Nevertheless, we would like to focus attention on the $4 / 33$ (12.12\%) patients from our study who had a diagnosis of non-calcific type of chronic pancreatitis (Figure 1) [1]. Pancreatic duct disruption was an indication for endotherapy in each of the 4 patients [1]. We find it difficult to explain the possible cause of pancreatic stent fragmentation in this group.

Recently in our medical center we completed a retrospective analysis of 451 procedures of endoscopic retrograde pancreatography (ERP) with stenting of the main pancreatic duct. The procedures were carried out between 2001 and 2016 in 166 patients (122 men, 44 women, mean age: $52.96 \pm 13.27$; range: $26-86$ ) with walled-off pancreatic necrosis and pancreatic duct disruption. What is interesting, intraductal pancreatic stent fragmentation was recognized during 2/451 (0.44\%) endoscopic procedures in 2/166 (1.2\%) patients only (Figure 2). In these 2 patients the fragments of broken pancreatic stents were removed with rattooth forceps.

The mentioned outcomes confirm that intraductal pancreatic stent fragmentation is in fact an extremely rare complication of pancreatic endotherapy. In this short article we aim to be sensitive to the fact that a broken pancreatic stent may also occur in patients undergoing endoscopic treatment of main pancreatic duct disruption, regardless of the diagnosis of chronic pancreatitis. We agree with the majority of authors [2-4] that endoscopic procedures should be attempted at centers experienced in retrieval of broken pancreatic stents, despite the lack of guidelines for treatment of intraductal pancreatic stent fragmentation. We have developed in our medical center the strategy of endoscopic treatment of intraductal pancreatic stent fragmentation [1], which we also use in the patients with a proximally migrated pancreatic stent. According to our

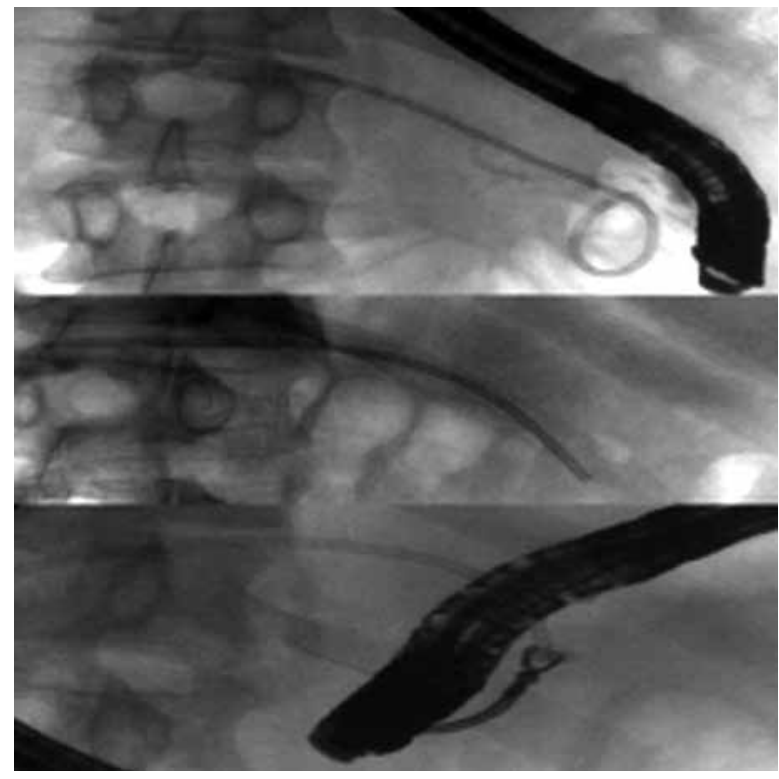

Figure 1. A 62-year-old patient with non-calcific chronic pancreatitis. The pancreatic broken stent in the pancreatic duct that was retrieved with rattooth forceps 

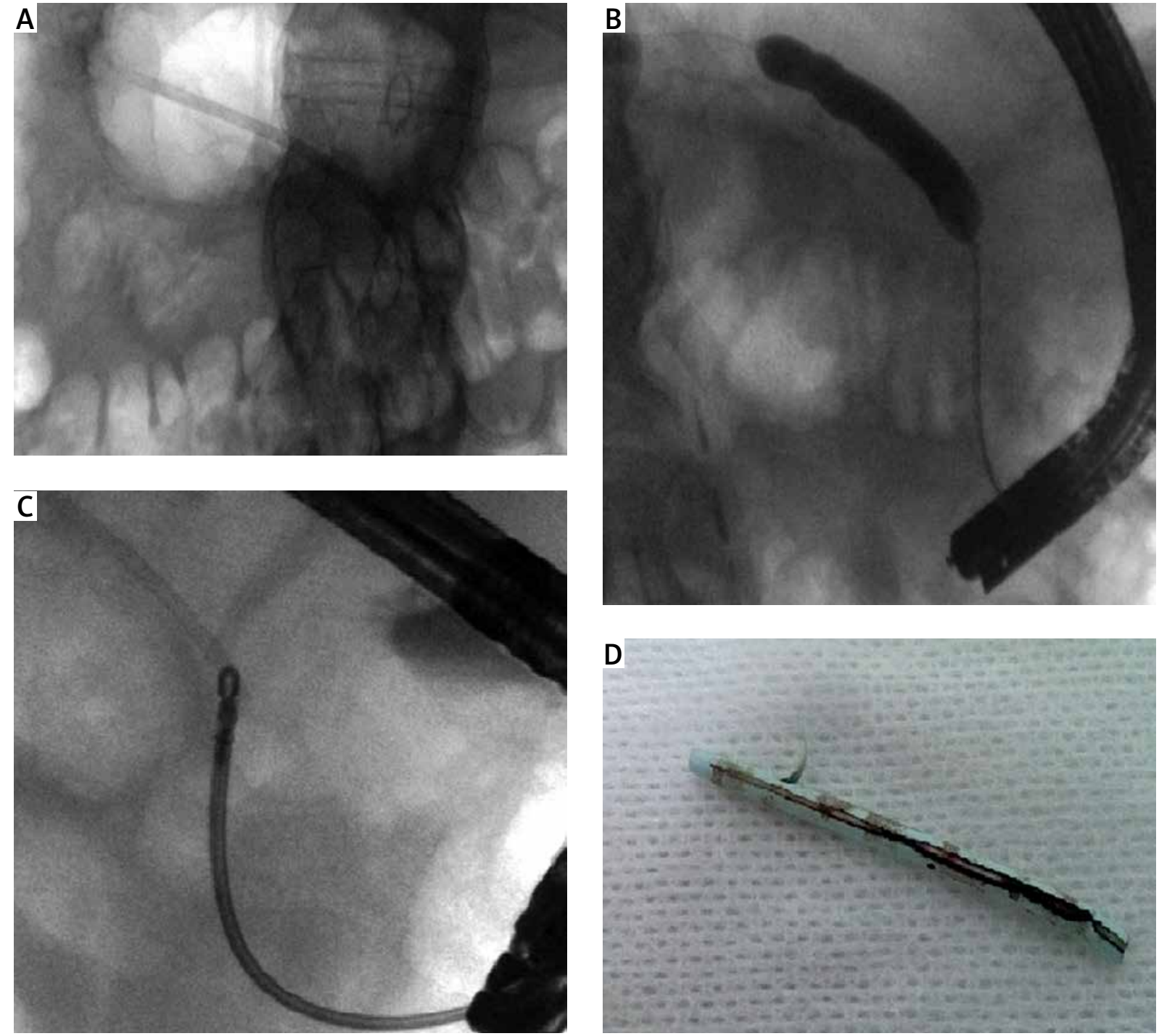

Figure 2. A 60-year-old man with intraductal pancreatic stent fragmentation during endoscopic treatment of pancreatic duct disruption and walled-off pancreatic necrosis as consequences of acute necrotizing pancreatitis. The fragments of broken stent were removed with forceps after dilation with a high pressure balloon

strategy, the first instrument used for retrieval of parts of the broken stent during each endoscopic procedure was rat-tooth grasping forceps (Figure 1). Retrieval of a broken pancreatic stent with forceps is a better method than removal using a basket or snare, because it allows the fragment's proximal tip to be grasped and the procedure is then less traumatic. Previous dilation of the main pancreatic duct with a high-pressure balloon enabled opening of the forceps (Figure 2), catching the broken stent fragment and its retrieval. Other instruments were used during the same endoscopic procedure only after a few unsuccessful attempts of removal of stent fragments from the lumen of the main pancreatic duct with forceps. However, in some cases it is necessary to use a polypectomy snare or Dormia basket, especially when the fragment of a broken stent is positioned in the pancreatic tail.

Another problem is the fact that patients with this type of complication are usually asymptomatic. In our opinion, an attempt of endoscopic removal of the migrated or broken pancreatic stent should be made prior to the considered surgical intervention, regardless of the lack of symptoms.

\section{Conflict of interest}

The authors declare no conflict of interest. 


\section{References}

1. Jagielski M, Smoczyński M, Jabłońska A, Adrych K. Endoscopic treatment of intraductal pancreatic stent fragmentation. Dig Endosc 2017; 29: 798-805.

2. Johanson JF, Schmalz MJ, Geenen JE. Incidence and risk factors for biliary and pancreatic stent migration. Gastrointest Endosc 1992; 38: 3416.

3. Kawaguchi Y, Lin JC, Kawashima Y, et al. Risk factors for migration, fracture, and dislocation of pancreatic stents. Gastroenterol Res Pract 2015; 2015: 365457.

4. Matsumoto K, Katanuma A, Maguchi H. Endoscopic removal technique of migrated pancreatic plastic stents. J Hepatobiliary Pancreat Sci 2014; 21: E34-40.

Received: 29.01.2018

Accepted: 21.03.2018 